\title{
Influência da temperatura e umidade na biologia de Rhodnius neglectus Lent, 1954 em laboratório (Hemiptera, Reduviidae,Triatominae)
}

\author{
Influence of the temperature and humidity on the biology of \\ Rhodnius neglectus Lent, 1954 in laboratory conditions
}

\author{
Dayse da Silva Rocha', José Jurberg' ${ }^{1}$, Rodolfo Ubaldo Carcavallo', Vanda Cunha1 e Cleber Galvão
}

\begin{abstract}
Resumo O objetivo do presente trabalho foi observar o desenvolvimento de R. neglectus em diferentes condições de temperatura e umidade. Três grupos foram formados:33/40 (33+/- $1^{\circ} \mathrm{C}$ e 40\% +/-5\% UR), 33/70 $\left(33+/-1^{\circ} \mathrm{C}\right.$ e $70 \%+1-5 \%$ UR), e 28/70 (28+/- $1^{\circ} \mathrm{C}$ e $70 \%+1-5 \%$ UR). Todos os grupos foram observados diariamente para avaliação do período de incubação dos ovos, período de desenvolvimento dos estádios ninfais, percentual de mortalidade por estádio, número de repastos realizados, e período de desenvolvimento da eclosão a muda imaginal. O desenvolvimento embrionário apresentou diferenças significativas em todos os tratamentos, o grupo 33/40 apresentou o menor período de incubação. O menor número de repastos foi observado no grupo 33/40 exceto para o primeiro estádio (grupo 28/70). O período de desenvolvimento ninfal foi significativamente reduzido no grupo 33/40; entretanto, o percentual de mortalidade deste grupo foi alto, e os insetos não conseguiram realizar a muda imaginal.
\end{abstract}

Palavras-chaves: Rhodnius neglectus. Triatominae. Doença de Chagas. Temperatura. Umidade.

Abstract The objective of the present paper was to study the development of $R$. neglectus in the laboratory under different conditions of temperature and humidity. Three groups were prepared: $33 / 40\left(33+/-1{ }^{\circ} \mathrm{C}\right.$ and $40 \%$ $+/-5 \%$ UR), $33 / 70\left(33+/-1{ }^{\circ} \mathrm{C}\right.$ and $70 \%+/-5 \%$ UR), and $28 / 70\left(28+/-1{ }^{\circ} \mathrm{C}\right.$ and $70 \%+/-5 \%$ UR). All these groups were observed daily to evaluate the eggs' incubation period, the development period of each instar nymph, the mortality rate of each stage, the frequency of feeding and the hatching-adult ecdysis period. The embryonic development showed significant differences between the treatment given, the group 33/40 presented the lowest incubation period. The lowest bloodmeals number was observed in the group 33/40 except for the first instar (group 28/70). The period of nymphal development was significantly reduced in the group 33/40, however, the mortality was high and the specimens did not reach the adult phase.

Key-words: Rhodnius neglectus. Triatominae. Chagas' disease. Temperature. Humidity.

O aquecimento global e seus possíveis impactos físicos e ecológicos, vem despertando o interesse de pesquisadores de diversas áreas; os impactos que as mudanças climáticas poderão ocasionar na saúde humana têm sido tema de debates entre especialistas da OMS ${ }^{1}$. No caso da doença de Chagas, as prováveis alterações seriam: modificações na distribuição geográfica das espécies; alterações no mecanismo de dispersão pelo vôo; incremento do número de repastos, aumentando a probabilidade de infecção/transmissão; aceleração do ciclo biológico e aumento da densidade populacional ${ }^{812}$. Rhodnius neglectus Lent, 1954, é uma espécie encontrada nos Estados da Bahia, Goiás, Maranhão, Mato Grosso, Minas Gerais, Paraná, Pernambuco e São Paulo, Brasil, entre $3^{\circ}$ e $25^{\circ}$ de latitude sul, desde o nível do mar até 700 m.s.n.m . É considerada silvestre mas, já foi encontrada nas habitações humanas no Estado de Goiás, juntamente com o T. sordida ${ }^{23}$. Em estudos realizados nas regiões norte e nordeste do Estado de São Paulo, e no Estado de Minas Gerais, foram encontradas colônias de $R$. neglectus em domicílios e peridomicílios 5 . Considera-se, ainda que em menor grau, capaz de colonizar ecótopos artifíciais, mesmo a distâncias apreciáveis dos focos naturais ${ }^{18}$. Esta espécie está entre as espécies que vem ocupando os nichos vagos, após o controle do T. infestans no Estado de Goiás ${ }^{27}$. No ambiente silvestre $R$. neglectus está associado a várias espécies de palmeiras como o babaçu e a macaubeira, utilizando como fonte alimentar aves, marsupiais,

\footnotetext{
1 Laboratório Nacional e Internacional de Referência em Taxonomia de Triatomíneos do Departamento de Entomologia do Instituto Oswaldo Cruz, Rio de Janeiro, RJ. Suporte Financeiro: CNPq e convênio FNS/FIOCRUZ no 123/97

Endereço para correspondência: Dra. Dayse da Silva Rocha. Av. Brasil 4365, Manguinhos, 21045-900 Rio de Janeiro RJ, Brasil.

Telefax:55 $212290-9339$

e-mail:galvao@gene.dbbm.fiocruz.br

Recebido para publicação em 8/9/2000.
} 
roedores e quirópteros ${ }^{10}$. No peridomicílio é encontrado principalmente em galinheiros ${ }^{321}$, e já foi encontrado naturalmente infectado pelo $T$. cuzi ${ }^{2}$. D'Alessandro ${ }^{13}$ infectou-o, experimentalmente, com T. rangeli, encontrando, posteriormente parasitos nas fezes e glândulas salivares e Diotaiuti et $a^{17}$ o encontraram naturalmente infectado por este parasito. Diferentes índices de infecção natural pelo $T$. cruzi têm sido encontrados e variam de lugar para lugar, devido provavelmente a circunstanciais contatos com fontes infectadas. Barretto et a/ observaram $4,4 \%$ de infecção pelo T. cruzi nos insetos capturados; dois anos mais tarde, esses autores encontraram um índice mais baixo $(2,5 \%)$. Índices de infecção bem superiores $(15,9 \%)$ foram encontrados, sugerindo que $R$. neglectus tenha importante papel na transmissão silvestre na periferia de Belo Horizonte, Minas Gerais ${ }^{15}$.

No presente trabalho, foi verificada a influência de diferentes associações de temperatura e umidade sobre o desenvolvimento embrionário e ninfal de $R$. neglectus; sobre o número de repastos sangüíneos realizados e sobre os percentuais de mortalidade.

\section{MATERIAIS E MÉTODOS}

Foram utilizados espécimes de $R$. neglectus provenientes de colônias mantidas em temperatura ambiente, com alimentação quinzenal em pombos (Columba livia), no insetário do Laboratório Nacional e Internacional de Referência em Taxonomia de Triatomíneos do Departamento de Entomologia do Instituto Oswaldo Cruz. Foram retiradas aleatoriamente da colônia cem ninfas de 5 ㅇ estádio para obtenção de adultos virgens. Após a muda imaginal foram formados 40 casais, alimentados semanalmente em pombos e mantidos em conjunto para obtenção de ovos. A partir das observações do período embrionário, foram formados 3 grupos distintos, mantidos em diferentes condições: $33 / 40$ (33 +/- $1^{\circ} \mathrm{C}$ e $40+/-5 \%$ de U.R.); 33/ $70\left(33+/-1{ }^{\circ} \mathrm{C}\right.$ e $70+/-5 \%$ de U.R.) e $28 / 70\left(28+/-1^{\circ} \mathrm{C}\right.$ e $70+/-5 \%$ de U.R.). Para cada grupo foram utilizados 100 ovos, oriundos de posturas realizadas no mesmo dia. Após a eclosão, os insetos de cada grupo foram transferidos para cristalizadores de vidro transparente ( $20 \mathrm{~cm}$ de altura e $20 \mathrm{~cm}$ de diâmetro) contendo no seu interior papel de filtro e tiras dobradas. A alimentação foi realizada em pombos imobilizados e oferecida semanalmente por um período de 3 horas. Os grupos foram mantidos em estufa B.O.D. com fotofase de 12 horas; no grupo 33/40 utilizou-se sílica gel para manutenção da umidade relativa em $40+/-5 \%$. Todos os grupos foram observados diariamente para avaliação do período de incubação dos ovos, do período de desenvolvimento de cada estádio, dos percentuais de mortalidade por estádio, do número de repastos realizados por estádio e do período de desenvolvimento da eclosão até a muda imaginal. Os resultados foram analisados estatisticamente pelos testes de MannWhitney e Kruskal-Wallis.

\section{RESULTADOS}

Período de incubação dos ovos. $R$. neglectus apresentou um período de desenvolvimento embrionário, bastante variável em todos os tratamentos. Ocorreram diferenças significativas entre os grupos $33 /$ $40,33 / 70$ e $28 / 70$, demonstrando a sensibilidade desta espécie aos tratamentos utilizados. O grupo $33 / 40$ foi o que apresentou o menor período de incubação variando de 9 a 16 dias, e o grupo 28/70 o que apresentou o maior período variando entre 12 e 18 dias. A influência da temperatura pôde ser observada na diminuição do período médio de incubação dos ovos dos grupos 33/ 40 e 33/70 ambos mantidos em temperatura mais alta (Tabela 1). Em todas as comparacõoes estatísticas entre os grupos estudados $(33 / 70 \times 33 / 40,33 / 70 \times 28 /$ 70 e 33/40 x 28/70) foram encontradas diferenças significativas $(p<0.01)$.

Número de repastos realizados por fase de desenvolvimento. O oferecimento semanal da alimentação e a manutenção dos espécimes que se recusavam a sugar, permitiram conhecer o número de repastos necessários para que um grupo de espécimes atingisse o estádio seguinte. $R$. neglectus apresentou um aumento crescente no número de repastos em relação às fases de desenvolvimento, e foram constatadas diferenças significativas em relação ao número de repastos realizados por estádio em diferentes comparações. O grupo $33 / 40$ foi o que apresentou o menor número de repastos em todas as fases de desenvolvimento observadas, (exceto no 1 o estádio) com médias de 1,$8 ; 2,3 ; 3,2 ; 3,8$ respectivamente, e o grupo $33 / 70$ o que necessitou de maior número (1으o ao 5 estádios) 1,9; 3,4; 4,1; 5,2; 6,2 respectivamente. Foram encontradas diferenças significativas em todos os tratamentos utilizados (Tabelas 2 e 3 ).

Período de desenvolvimento ninfal. O período de desenvolvimento ninfal foi nitidamente diferenciado de acordo com as temperaturas e umidades utilizadas. O menor período médio de desenvolvimento em todas as fases foi observado no grupo $33 / 40 \mathrm{com}$ médias do 1 o ao 4 o estádio de 12,$5 ; 16,6 ; 19,6$ e 22,8 respectivamente. $O$ maior período médio, observado em todas as fases de desenvolvimento foi verificado no grupo $33 / 70$ com os seguintes valores do $1^{\circ}$ ao $5^{\circ}$ estádios 14,4;24,7;32,3; 38; e 54,8. Apesar de nenhum espécime ter realizado a muda imaginal, o grupo 33/40 foi o que registrou o menor período médio total de desenvolvimento (da eclosão à muda para o 5 o estádio) 71,7 dias, quando comparado ao mesmo período este valor é $29,5 \%$ inferior ao observado no grupo 28/70 (101 
Tabela 1 - Período (em dias) de incubação dos ovos de Rhodnius neglectus Lent, 1954 submetidos a diferentes temperaturas e umidades.

\begin{tabular}{ccccccc}
\hline $\begin{array}{c}\text { Temperatura } \\
{ }^{\circ} \mathrm{C}\end{array}$ & $\begin{array}{c}\text { Umidade } \\
\%\end{array}$ & Mín & Máx & $\mathrm{X}$ & $\mathrm{S}$ & $\mathrm{S}^{2}$ \\
\hline $33+/-1$ & $70+/-5$ & 11 & 17 & 12,89 & 2,36 & 5,58 \\
$33+/-1$ & $40+/-5$ & 9 & 16 & 11,28 & 2,46 & 6,06 \\
$28+/-1$ & $70+/-5$ & 12 & 18 & 14,77 & 2,14 & 4,61 \\
\hline
\end{tabular}

\begin{tabular}{|c|c|c|c|c|c|c|c|}
\hline Estádio & $\begin{array}{l}\text { Temperatura } \\
{ }^{\circ} \mathrm{C}\end{array}$ & $\begin{array}{l}\text { Umidade } \\
\%\end{array}$ & Mín & Máx & $x$ & $S$ & $\mathrm{~S}^{2}$ \\
\hline $1 \stackrel{0}{9}$ & 33 & 40 & 1 & 5 & 1,81 & 0,87 & 0,75 \\
\hline $2^{\circ}$ & 33 & 40 & 1 & 5 & 2,35 & 1,42 & 2,02 \\
\hline $3^{0}$ & 33 & 40 & 2 & 6 & 3,26 & 1,04 & 1,09 \\
\hline $4^{\circ}$ & 33 & 40 & 2 & 7 & 3,83 & 1,83 & 3,36 \\
\hline $5^{\circ}$ & 33 & 40 & - & - & - & - & - \\
\hline$\overline{10}$ & 33 & 70 & 1 & 4 & 1,98 & 0,65 & 0,43 \\
\hline $2^{0}$ & 33 & 70 & 1 & 5 & 3,43 & 0,93 & 0,87 \\
\hline $3^{\circ}$ & 33 & 70 & 2 & 7 & 4,11 & 1,56 & 2,45 \\
\hline $4^{\circ}$ & 33 & 70 & 2 & 8 & 5,28 & 1,77 & 3,14 \\
\hline $5^{\circ}$ & 33 & 70 & 3 & 11 & 6,20 & 2,94 & 8,70 \\
\hline $1^{\circ}$ & 28 & 70 & 1 & 5 & 1,57 & 0,92 & 0,85 \\
\hline $2^{0}$ & 28 & 70 & 1 & 6 & 3,17 & 0,84 & 0,71 \\
\hline $3^{0}$ & 28 & 70 & 1 & 6 & 3,50 & 1,12 & 1,25 \\
\hline $4^{\circ}$ & 28 & 70 & 2 & 9 & 4,30 & 1,44 & 2,08 \\
\hline $5^{\circ}$ & 28 & 70 & 3 & 8 & 4,27 & 1,25 & 1,57 \\
\hline
\end{tabular}

(-) Os insetos não completaram o 5ำ estádio.

Tabela 3 - Comparações estatísticas do número de repastos realizados em cada fase de desenvolvimento de Rhodnius neglectus, Lent, 1954 mantido em diferentes condições de temperatura e umidade.

\begin{tabular}{lcrccc}
\hline Tratamento & 1e estádio & 2estádio & 3o estádio & 4 estádio & 5 estádio \\
\hline $33 / 70 \times 33 / 40$ & $\mathrm{NS}$ & $\mathrm{P}<0,01$ & $\mathrm{NS}$ & $\mathrm{NS}$ & - \\
$33 / 70 \times 28 / 70$ & $\mathrm{P}<0,01$ & $\mathrm{P}<0,05$ & $\mathrm{NS}$ & $\mathrm{P}<0,05$ & NS \\
$33 / 40 \times 28 / 70$ & $\mathrm{P}<0,05$ & $\mathrm{P}<0,01$ & $\mathrm{NS}$ & $\mathrm{NS}$ & - \\
\hline
\end{tabular}

$\mathrm{NS}=$ diferenças não significativas

dias) e 34,5\% ao registrado para o grupo 33/70 (109 dias). Foram observadas diferenças significativas entre os grupos $33 / 70 \times 33 / 40$ e $33 / 40 \times 28 / 70$. Quando comparou-se os grupos com a mesma umidade (33/70 e 28/70) não foram registradas diferenças significativas em nenhum dos estádios (Tabelas 4 e 5).

Percentual de mortalidade por fase de desenvolvimento. Os percentuais de mortalidade em cada fase de desenvolvimento estão representados na Figura 1, variaram em relação aos estádios de desenvolvimento, e aos tratamentos utilizados. Para o grupo $33 / 70$ os maiores percentuais foram observados no $1^{\circ}$ estádio $(55 \%)$ e $5^{\circ}$ estádio $(64,2 \%)$, no grupo $28 / 70$ o maior percentual de mortalidade foi registrado para 0 $2^{\circ}$ estádio $(29,8 \%)$, e nos insetos mantidos a $33 / 40$ o $4^{\circ}$ estádio foi o que apresentou maior índice de mortalidade.

\section{DISCUSSÃO}

Desenvolvimento embrionário. No presente trabalho, o grupo $33 / 40$ foi o que apresentou menor período de incubação dos ovos, e o grupo 28/70 o que apresentou maior período. Dias ${ }^{14}$ chamava atenção para as diferenças no período de desenvolvimento embrionário de $R$. neglectus, verificando que em ovos postos em setembro (Rio de Janeiro) o período de incubação variou de 20 a 23 dias, e nas posturas realizadas em março, entre 15 e 17 dias. Estes valores registrados no mês de março são próximos aos valores 
Tabela 4 - Período de desenvolvimento (em dias), das ninfas de Rhodnius neglectus Lent, 1954 mantidos em diferentes condições de temperatura e umidade

\begin{tabular}{|c|c|c|c|c|c|c|c|c|}
\hline Estádio & $\begin{array}{l}\text { Temperatura } \\
{ }^{\circ} \mathrm{C}\end{array}$ & $\begin{array}{l}\text { Umidade } \\
\%\end{array}$ & Mín & Máx & $x$ & $S$ & $S^{2}$ & $\mathrm{~N}$ \\
\hline 10 & 33 & 40 & 7 & 25 & 12,59 & 4,34 & 18,87 & 74 \\
\hline $2^{\circ}$ & 33 & 40 & 8 & 29 & 16,69 & 5,67 & 32,21 & 39 \\
\hline $3^{\circ}$ & 33 & 40 & 13 & 38 & 19,63 & 5,88 & 34,69 & 19 \\
\hline $4^{\circ}$ & 33 & 40 & 11 & 39 & 22,83 & 10,04 & 100,96 & 06 \\
\hline $5^{\circ}$ & 33 & 40 & - & - & - & - & - & - \\
\hline $1 \stackrel{0}{9}$ & 33 & 70 & 7 & 32 & 14,46 & 4,98 & 24,81 & 54 \\
\hline $2^{\circ}$ & 33 & 70 & 11 & 38 & 24,76 & 8,41 & 70,87 & 39 \\
\hline $3^{\circ}$ & 33 & 70 & 17 & 49 & 32,22 & 10,36 & 10,36 & 19 \\
\hline $4^{\circ}$ & 33 & 70 & 13 & 90 & 38,00 & 20,08 & 403,38 & 06 \\
\hline $5^{\circ}$ & 33 & 70 & 27 & 92 & 54,80 & 23,73 & 563,20 & 05 \\
\hline $1^{\circ}$ & 28 & 70 & 9 & 39 & 14,68 & 6,16 & 37,94 & 97 \\
\hline $2^{0}$ & 28 & 70 & 9 & 55 & 24,10 & 8,56 & 73,31 & 68 \\
\hline $3^{0}$ & 28 & 70 & 14 & 49 & 27,54 & 8,77 & 77,06 & 55 \\
\hline $4^{\circ}$ & 28 & 70 & 20 & 70 & 34,80 & 10,85 & 117,84 & 46 \\
\hline $5^{\circ}$ & 28 & 70 & 23 & 76 & 38,28 & 11,26 & 126,85 & 36 \\
\hline
\end{tabular}

(-) Os insetos não completaram o 5ํestádio.

Tabela 5 - Comparações estatísticas do período de desenvolvimento (em dias), das ninfas de Rhodnius neglectus Lent, 1954 mantidos em diferentes condições de temperatura e umidade.

\begin{tabular}{lccccc}
\hline Tratamento & 1 estádio & 2 2 estádio & 3 estádio & 4 estádio & 5 estádio \\
\hline $33 / 70 \times 33 / 40$ & $\mathrm{P}<0,05$ & $\mathrm{P}<0,01$ & $\mathrm{P}<0,01$ & $\mathrm{NS}$ & - \\
$33 / 70 \times 28 / 70$ & $\mathrm{NS}$ & $\mathrm{NS}$ & $\mathrm{NS}$ & $\mathrm{NS}$ & $\mathrm{NS}$ \\
$33 / 40 \times 28 / 70$ & $\mathrm{NS}$ & $\mathrm{P}<0,01$ & $\mathrm{P}<0,01$ & $\mathrm{P}<0,05$ & - \\
\hline
\end{tabular}

$\mathrm{NS}=$ diferenças não significativas

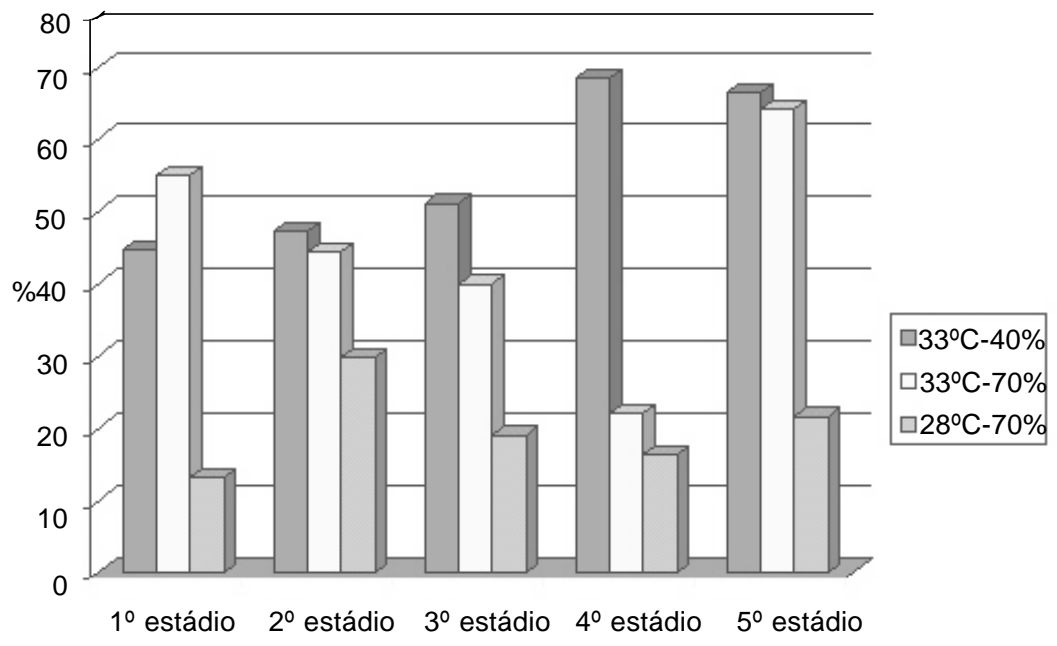

Figura 1- Percentuais de mortalidade das ninfas de Rhodnius neglectus Lent, 1954 mantidas em diferentes condições de temperatura e umidade.

máximos observados no presente trabalho, para os grupos mantidos em temperatura mais alta, 16 dias (33/ 40) e 17 dias (33/70). Gómez-Núnez ${ }^{19}$ trabalhando com R. prolixus observou que apesar de ocorrerem eclosões em temperaturas constantes entre 16 e $34^{\circ} \mathrm{C}$, geralmente só são observados percentuais em torno de $100 \%$ quando os ovos são mantidos em temperaturas de 20 a $29^{\circ} \mathrm{C}$. Em $R$. neglectus foram 
constatadas diferenças significativas entre todos os tratamentos, os grupos mantidos em temperaturas mais altas $\left(33^{\circ} \mathrm{C}\right)$ foram os que apresentaram menor período de desenvolvimento embrionário. $O$ período médio de incubação registrado no presente trabalho $(11,2)$ quando submetido ao tratamento $33 / 40$ é um dos mais curtos conhecidos até o momento para esta espécie, aproximando-se ao observado por Lima \& Costa $^{22}$ que ao estudarem alguns aspectos da biologia de $R$. neglectus em condições de laboratório e sua evolução em temperatura ambiente, observaram um período médio de incubação de 12 dias. No presente trabalho observamos uma nítida correlação entre as temperaturas mais altas, e a diminuição do período embrionário.

Números de repastos realizados em cada fase do desenvolvimento. Para cada espécie, existe uma freqüência de alimentação ótima e uma freqüência crítica, onde os parâmetros vitais começam a ser afetados. O número de repastos realizados tem importância epidemiológica, já que quantos mais contatos ocorrerem entre vetores e hospedeiros, maior será a probabilidade de infecção ou transmissão do T. cruzi. Entretanto, o número de repastos necessários para que ocorram as ecdises não é mencionado pela maioria dos autores, sendo geralmente relatados apenas os intervalos entre os repastos.

O aumento de temperatura associado à baixa umidade relativa reduz ainda mais o período de desenvolvimento. Seria então, esperado um aumento da freqüência de repastos sangüíneos, como forma de compensar a perda de água pela associação entre a alta temperatura e baixa umidade ${ }^{8}$. Entretanto, no presente trabalho $R$. neglectus diminuiu o número de repastos quando submetido a alta temperatura e baixa umidade. $O$ oferecimento semanal da alimentação permitiu avaliar o número de repastos necessários à ocorrência de ecdise em cada estádio, e por não desprezarmos os insetos que se recusaram a sugar, as variações no número de repastos realizados foram bastante heterogêneas, sendo conseqüência de diferenças intra-específicas. Segundo PerlowagoraSzumlewicz ${ }^{25}$ as ninfas de 1 o estádio tendem a se alimentar entre $02^{\circ}$ e 0 6 dia após a eclosão. Em $R$. neglectus observamos que o grupo $33 / 40$ foi o que apresentou menor número de repastos para que os insetos atingissem o 5o estádio, e o 33/70 o que realizou maior número no mesmo período.

Período de desenvolvimento ninfal. Buxton ${ }^{6} 7$ observou a duração total do ciclo de vida de $R$. prolixus de 99 dias a $24^{\circ} \mathrm{C}$ e 73 dias a $30^{\circ} \mathrm{C}$, concluindo que em temperaturas ao redor de $42^{\circ} \mathrm{C}$ encontra-se o limite térmico superior do gênero Rhodnius. Okasha ${ }^{24}$ trabalhando com $R$. prolixus, considerou como subletal a temperatura de $36,5^{\circ} \mathrm{C}$. A temperatura de $20^{\circ} \mathrm{C}$, foi apontada como a temperatura mínima necessária para alimentação, dispersão e reprodução de espécies na Argentina ${ }^{11}$. No presente trabalho observou-se que os espécimes do grupo 33/40, foram os que apresentaram o menor período de desenvolvimento ninfal em todos os estádios observados, redução esta que representou um encurtamento do ciclo de aproximadamente $32 \%$. Em relação ao período de desenvolvimento ninfal total o grupo $28 / 70$ foi o único onde surgiram adultos, nos demais tratamentos os insetos atingiram o 5o estádio mas não conseguiram chegar a fase adulta, morrendo neste estádio, exceto no grupo 33/40 em que um espécime chegou a fase adulta morrendo durante o processo de muda. Diotaiuti \& Dias $^{16}$ mantiveram $R$. neglectus em insetário em temperatura de $27+/-2^{\circ} \mathrm{C}$ e U.R. de $70 \%$ utilizando camundongos e pombos como fonte alimentar. O desenvolvimento médio foi de 150,3 dias para os insetos alimentados em camundongos, período inferior ao obtido para os insetos alimentados em pombos, porém, superior ao obtido no tratamento $28 / 70$ do presente trabalho. A temperatura de $33^{\circ} \mathrm{C}$ foi limitante neste experimento independentemente da umidade, já que quando submetida a $40 \%$ e $70 \%$ respectivamente, não ocorreu um número significativo de mudas imaginais. $R$. neglectus não demonstrou boa capacidade de adaptação a estas condições em laboratório, apesar desta temperatura não ser considerada alta por vários autores. Heitzmann-Fontenelle ${ }^{20}$, estudando a bionomia de $R$. neglectus, considerou o desenvolvimento desta espécie rápido, tendo um período de desenvolvimento total de um ano e um mês a três anos e dois meses. Quando comparou-se estes resultados com os obtidos no presente trabalho, verificou-se que este período é aproximadamente 3 vezes superior ao obtido no tratamento $33 / 70$, que foi o grupo que apresentou o período de desenvolvimento mais longo. Até o momento os períodos de desenvolvimento observados para $R$. neglectus, em todos os tratamentos utilizados no presente trabalho foram os mais curtos registrados na literatura.

Percentuais de mortalidade. Os percentuais de mortalidade mostraram-se distintos entre os tratamentos utilizados nos diferentes estádios, os maiores percentuais de mortalidade foram registrados no grupo $33 / 40$, exceto para o 1 estádio onde o maior percentual foi o observado no grupo 33/70. As taxas de mortalidade dos triatomíneos geralmente são mais elevadas no $1^{\circ} \mathrm{e}$ 5 estádios; no 1ㅇ geralmente pela dificuldade na realização do primeiro repasto, e no $5^{\circ}$ estádio principalmente pela ocorrência de ecdises defeituosas e interrupções na muda imaginal, que é mais complexa que nas demais fases. No presente trabalho, entretanto, os maiores percentuais foram registrados no 4ㅇ e $5^{\circ}$ estádios, nos grupos 33/40 e 33/70; no grupo 28/70 esses percentuais apresentaram valores nitidamente inferiores. Foi observada uma relação diretamente proporcional entre a temperatura e as porcentagens de mortalidade ninfal em $T$. infestans, que oscila entre 3,4\% a $15^{\circ} \mathrm{C}$ e $36 \%$ a $35^{\circ} \mathrm{C}^{26}$. Durante todo o experimento a maioria das mortes observadas foram decorrentes de problemas na ecdise, onde os insetos de todos os estádios iniciavam o processo de muda, mas morriam sem conseguir livrar-se da exúvia. Os resultados obtidos suportam que, temperaturas elevadas associadas ou 
não a baixas umidades relativas, podem acelerar o ciclo ninfal de $R$. neglectus, entretanto, apesar deste encurtamento, esta espécie apresentou dificuldades na realização da muda imaginal, não sendo recomendada a utilização desta temperatura para manutenção de colônias em laboratório. Neste experimento, foram utilizadas condições extremas de temperatura $\left(33^{\circ} \mathrm{C}\right)$ e umidade relativa (40\%), e também a temperatura e umidade consideradas ótimas para maioria das espécies $\left(28^{\circ} \mathrm{C}\right.$ e $\left.70 \%\right)$. Trabalhar com esses tratamentos distintos nos permitiu avaliar a influência dessas condições ambientais em alguns parâmetros biológicos de $R$. neglectus, e analisar sua capacidade de adaptação frente às variações destes importantes parâmetros ambientais.

\section{AGRADECIMENTO}

A Octavio AF Presgrave pelas análises estatísticas.

\section{REFERÊNCIAS BIBLIOGRÁFICAS}

1. Ando M, Carcavallo RU, Epstein PR. Climate change and human health In:McMichael AM, Haines A, Sloof R, Kovats S (eds) World Health Organization, Geneva, Switzerland, p.154-167, 1996.

2. Barretto MP. Estudos sobre reservatórios e vetores silvestres do Trypanosoma cruzi, XVII: Contribuição para o estudo dos focos naturais da tripanosomíase americana, com especial referência à região nordeste do Estado de São Paulo. Revista da Sociedade Brasileira de Medicina Tropical 1: 23, 1967.

3. Barretto MP, Albuquerque RDR, Funayama GK. Estudos sobre reservatórios e vectores silvestres do Trypanosoma cruzi. XXXVI. Investigações sobre triatomíneos de palmeiras no Município de Uberaba, Minas Gerais, Brasil. Revista Brasileira de Biologia 29:577-588, 1969.

4. Barretto MP, Siqueira AF, Ferriolli-Filho F, Carvalheiro JR. Estudos sôbre reservatórios e vectores silvestres do Trypanosoma cruzi. XI - Observações sôbre um foco natural da tripanossomose americana no Município de ribeirão Prêto, São Paulo. Revista do Instituto de Medicina Tropical de São Paulo 8: 103-112, 1966.

5. Barretto MP, Siqueira AF, Ferriolli-Filho F, Carvalheiro JR. Estudos sôbre reservatórios e vectores silvestres do Trypanosoma cruzi. XXIII - Observações sôbre criadouros do Rhodnius neglectus Lent,1954 em biótopos artificiais (Hemiptera- Reduviidae). Revista do Instituto de Medicina Tropical de São Paulo 10: 163170, 1968.

6. Buxton PA. The biology of a blood sucking bug Rhodnius prolixus. Transactions of Entomological Society of London 78: 227-236, 1930.

7. Buxton PA. The relation of adult Rhodnius prolixus (Reduviidae, Rhynchota) to atmospheric humidity. Parasitology 24:429-439, 1932.

8. Carcavallo RU. Climatic factors related to Chagas disease transmission. Memórias do Instituto Oswaldo Cruz 94 (supl:) 367369, 1999

9. Carcavallo RU, Curto de Casas SI, Sherlock IA, Galíndez-Girón I, Jurberg J, Galvão C, Mena Segura CA. Geographical distribution and altilatitudinal dispersion. In: Carcavallo RU, Galíndez- Girón I, Jurberg J, Lent $\mathrm{H}$ (orgs) Atlas of Chagas' disease vectors in the Americas, Vol. III, Editora FIOCRUZ, Rio de Janeiro, Brasil, p. 747-792, 1999.

10. Carcavallo RU, Franca Rodriguez ME, Salvatella R, Curto de Casas SI, Sherlock I, Galvão C, Rocha DS, Galíndez-Girón I, Otero Arocha MA, Martínez A, Rosa J A, Canale D, Farr TH, Barata JMS. Habitats and related fauna. In: Carcavallo RU,
Galíndez- Girón I, Jurberg J, Lent H (orgs) Atlas of Chagas' disease vectors in the Americas, Vol. II, Editora FIOCRUZ, Rio de Janeiro, Brasil, p. 561-600, 1998.

11. Curto de Casas $\mathrm{SI}$, Carcavallo RU. Limites del triatomismo en la Argentina. I: Patagônia. Chagas 1:35-40, 1984.

12. Curto de Casas SI, Carcavallo RU, Galíndez-Girón I, Burgos JJ. Bioclimatic factors and zones of life. Fatores bioclimáticos e zonas de vida. In: Carcavallo RU, Galíndez-Girón I, Jurberg J, Lent H. (orgs) Atlas of Chagas' disease vectors in the Americas. Atlas dos vetores da doença de Chagas nas Américas, Vol. III, Editora FIOCRUZ, Rio de Janeiro, Brasil, p. 793-838, 1999.

13. D'Alessandro A. New experimental vectors of Colombian Trypanosoma rangeli. Journal Medical Entomology 9: 187-195, 1972.

14. Dias E. Nota sôbre o tempo de evolução de algumas espécies de triatomíneos em Laboratório. Revista Brasileira de Biologia 15:157-158, 1955.

15. Diotaiuti L, Dias JCP. Ocorrência e biologia do Rhodnius neglectus Lent, 1954 em macaubeiras da periferia de Belo Horizonte, Minas Gerais. Memórias do Instituto Oswaldo Cruz 79: 293-301, 1984.

16. Diotaiuti L, Dias JCP. Estudo comparativo do ciclo evolutivo de Rhodnius neglectus alimentados em pombos ou camundongos. Revista da Sociedade Brasileira de Medicina Tropical 20: 95100, 1987.

17. Diotaiuti L, Silveira AC, Elias M, Steindel M. The possibility of occurrence of Trypanosoma rangeli in the State of Tocantins, Brazil. Memórias do Instituto Oswaldo Cruz 87: 451,1992.

18. Forattini OP, Ferreira OA, Rocha e Silva EO, Rabello EX. Aspectos ecológicos da tripanossomíase Americana. XV Desenvolvimento, variação e permanência de Triatoma sordida, Panstrongylus megistus e Rhodnius neglectus em ecótopos artificiais. Revista de Saúde Pública 13:220-234, 1979.

19. Goméz-Núñez JC. Mass rearing of Rhodnius prolixus . Bulletin of World Health Orgazization 31:565-567,1964.

20. Heitzmann-Fontenelle TJ. Bionomia comparativa de triatomíneos. VII - Rhodnius neglectus Lent, 1954 (Hemiptera, Reduviidae). Memórias do Instituto Butantan 47/48:183-188, 1983/1984.

21. Lent $\mathrm{H}$, Wygodzinsky P. Revision of the Triatominae (Hemiptera, Reduviidae), and their significance as vectors of Chagas' disease. Bulletin of the American Museum of Natural History 163: 123520,1979

22. Lima JD, Costa HMA. Alguns aspectos da biologia do Rhodnius neglectus, 1954, Hemiptera-Triatominae, em condições de 
laboratório. V- Evolução em temperatura ambiente. Arquivos da Escola de Veterinária da Universidade de Minas Gerais 22:151158, 1970.

23. Mello DA. Biology of Triatominae (Reduviidae- Hemiptera) from North of Formosa Country (Goiás-Brazil) 2. Length of life cycle of Rhodnius neglectus Lent, 1954. Revista da Sociedade Brasileira de Medicina Tropical 11:63-66, 1977.

24. Okasha AYK. Effects of sub-lethal high temperature on an insect Rhodnius prolixus (Stal). I Induction of the delayed moulting and defects. Experimental Biology 48: 455-463, 1968.
25. Perlowagora-Szumlewicz A. Laboratory colonies of Triatominae, biology and population dynamics. In: New Approaches in American Trypanosomiasis Research, Proceedings of an International Symposium. Pan American Health Organization Scientific Publication nr 318, Belo Horizonte, Brazil, p. 63-82, 1975.

26. Schofield CJ. The role of blood intake in density regulation of populations of Triatoma infestans (Klug) (Hemiptera: Reduviidae). Bulletin of Entomological Research 72: 617- 29,1982.

27. Silveira AC, Rezende DF. Epidemiologia da transmissão vetorial da doença de Chagas no Brasil. Revista da Sociedade Brasileira de Medicina Tropical 27(supl III):11-22, 1994. 